\title{
Depressão endogâmica na produção de leite no dia do controle de bovinos Gir leiteiro
}

\author{
Rodrigo Junqueira Pereira(1), Mário Luiz Santana Júnior ${ }^{(1)}$, Denise Rocha Ayres ${ }^{(1)}$, \\ Annaiza Braga Bignardi(1) e Aníbal Eugênio Vercesi Filho(2)
}

\begin{abstract}
(1)Universidade Federal de Mato Grosso, Instituto de Ciências Agrárias e Tecnológicas, Grupo de Melhoramento Animal de Mato Grosso, Rodovia MT-270, Km 06, Campus Rondonópolis, CEP 78735-001 Rondonópolis, MT, Brasil. E-mail: rodjunper@gmail.com, 10mario@gmail.com, d.ayres@ig.com.br, annaizabb@hotmail.com (2)Agência Paulista de Tecnologia dos Agronegócios, Instituto de Zootecnia, Rua Heitor Penteado, no 56, Centro, CEP 13460-000 Nova Odessa, SP, Brasil. E-mail: anibal@iz.sp.gov.br
\end{abstract}

Resumo - O objetivo deste trabalho foi estimar o efeito da endogamia sobre a produção de leite e sua persistência durante a lactação, em bovinos Gir leiteiro, bem como determinar o impacto de se considerar ou não o efeito da endogamia na avaliação genética dessa característica. Utilizaram-se 89.490 registros de produção no dia do controle, de 11.675 vacas em primeira lactação. O coeficiente e o incremento de endogamia individuais e o número equivalente de gerações completas de cada indivíduo foram computados. A depressão por endogamia foi estimada pela inclusão do efeito do incremento individual de endogamia, em um modelo de regressão aleatória. As soluções para a depressão por endogamia, de acordo com a classe de incremento individual de endogamia e a quinzena da lactação, variaram entre -1,238 e - $0,135 \mathrm{~kg}$, o que indica a redução da produção de leite com o aumento da endogamia. Em níveis mais elevados, a endogamia reduziu a persistência da produção de leite. A inclusão do efeito da endogamia no modelo de avaliação genética não afetou as estimativas de parâmetros genéticos e, praticamente, não alterou a predição dos valores genéticos e a classificação dos animais. Pode-se incluir o efeito da endogamia no modelo estatístico para a avaliação genética da característica produção de leite.

Termos para indexação: endogamia, parâmetros genéticos, persistência da produção de leite, regressão aleatória.

\section{Inbreeding depression in test-day milk yield of Gyr dairy cattle}

\begin{abstract}
The objective of this work was to estimate the effect of inbreeding on milk yield and its persistency during lactation, in Gyr dairy cattle, as well as to determine the impact of considering or not the effect of inbreeding on the genetic evaluation of this trait. Test-day records $(89,490)$ for milk yield from 11,675 dairy cows at first lactation were used. The individual inbreeding coefficient, the individual increase of inbreeding, and the number of equivalent complete generations of each individual were computed. Inbreeding depression was estimated by including the effect of individual increase of inbreeding in a random regression model. The solutions for inbreeding depression, according to the class of individual increase of inbreeding and stage of lactation, ranged between -1.238 and $-0.135 \mathrm{~kg}$, which indicates a reduction of milk yield with the increase of inbreeding. At higher levels of inbreeding, the persistence of milk yield was reduced. The inclusion of the inbreeding effect in the genetic evaluation model did not affect the genetic parameter estimates, with practically no change of the predicted breeding values and rank of animals. The inbreeding effect can be included in the statistical model for the genetic evaluation of milk yield.
\end{abstract}

Index terms: inbreeding, genetic parameters, persistence of milk yield, random regression.

\section{Introdução}

A raça Gir Leiteiro representa um importante material genético para a produção de leite nos trópicos. Isto decorre de suas características físicas e fisiológicas específicas, comuns também a outros zebuínos, que permitem aos animais serem mais adaptados aos sistemas de produção em pasto, particularmente aos desafios relacionados às altas temperaturas, à elevada umidade e à presença de endo- e ectoparasitas (Hansen, 2004; Jonsson, 2006).

Outro fator igualmente importante para o sucesso desta raça no Brasil é o trabalho de melhoramento genético para obtenção de aptidão leiteira, realizado pelos criadores. A Associação Brasileira dos Criadores de Gir Leiteiro (ABCGIL), juntamente com a 
Associação Brasileira dos Criadores de Zebu (ABCZ) e a Embrapa Gado de Leite, desenvolvem há 30 anos o Programa Nacional de Melhoramento do Gir Leiteiro (PNMGL), em que o principal objetivo é identificar, por meio de teste de progênie, touros geneticamente superiores quanto às características de produção, conformação e manejo (Panetto et al., 2015). O processo de seleção realizado pelos diferentes criadores é orientado pelas avaliações genéticas de touros e vacas divulgadas uma vez ao ano, que são realizadas tendo por base o modelo animal e o melhor preditor linear não viesado (BLUP). Neste procedimento, a acurácia dos valores genéticos preditos pode ser aumentada por meio da inclusão de informações de parentes; no entanto, há maior probabilidade de seleção simultânea de animais aparentados, o que causa aumento da taxa de endogamia $(\Delta \mathrm{F})$ e perda de variabilidade genética (Woolliams et al., 2015). Como consequência, o processo de melhoria do desempenho dos animais quanto às características de importância econômica, como a produção de leite e seus componentes, pode sofrer interferência negativa da depressão endogâmica, definida por Falconer \& Mackay (1996) como o declínio do valor fenotípico de uma característica, como consequência direta da endogamia.

Apesar de a estimação da depressão endogâmica em características de interesse econômico, nas populações de animais domésticos, ser realizada principalmente por meio da inclusão do coeficiente de endogamia do indivíduo $\left(\mathrm{F}_{\mathrm{i}}\right)$ como covariável em um modelo padrão de avaliação genética, González-Recio et al. (2007) sugeriram o uso do incremento individual de endogamia $\left(\Delta \mathrm{F}_{\mathrm{i}}\right)$, em substituição ao $\mathrm{F}_{\mathrm{i}}$, pois $\Delta \mathrm{F}_{\mathrm{i}}$, é mais adequado, por considerar a disparidade de conhecimento da genealogia entre os animais e, consequentemente, permitir uma estimativa mais acurada da depressão endogâmica.

Poucos estudos foram realizados com o objetivo de estimar a depressão endogâmica em características de zebuínos leiteiros e, somente um destes, realizado com dados de um rebanho da raça Guzerá, considerou o $\Delta \mathrm{F}_{\mathrm{i}}$ em substituição ao $\mathrm{F}_{\mathrm{i}}$ nas análises (Panetto et al., 2010). Nenhum desses, no entanto, avaliou o efeito da endogamia sobre a persistência da produção de leite durante a lactação.

O objetivo deste trabalho foi estimar a depressão por endogamia na produção de leite e sua persistência durante a lactação, em bovinos leiteiros da raça Gir, bem como determinar qual seria o impacto de se considerar ou não o efeito da endogamia sobre a avaliação genética dessa característica.

\section{Material e Métodos}

O banco de dados para a realização deste estudo foi disponibilizado pela ABCGIL, com inclusão dos registros genealógicos e de produção de leite do dia do controle (PLDC) de primeiras lactações de vacas da raça Gir. Tais informações provieram de rebanhos localizados em diversos estados do Brasil, participantes do programa oficial de coleta de dados do PNMGL.

As análises foram conduzidas em três etapas. $\mathrm{Na}$ primeira etapa, o arquivo de genealogia foi analisado com auxílio do programa RelaX2 (Strandén \& Vuori, 2006). Os registros genealógicos passaram por análise de consistência, para a eliminação de quaisquer possíveis erros. Em seguida, computaram-se o coeficiente de endogamia do indivíduo $\left(\mathrm{F}_{\mathrm{i}}\right)$, incremento individual de endogamia $\left(\Delta \mathrm{F}_{\mathrm{i}}\right)$ e o número equivalente de gerações completas (ECG) de cada indivíduo. O ECG para cada animal foi obtido pela soma, para todos os ancestrais conhecidos, dos termos $0,5^{\mathrm{n}}$, em que $\mathrm{n}$ é o número de gerações que separam o indivíduo de cada um dos seus ancestrais conhecidos. Os $\Delta \mathrm{F}_{\mathrm{i}}$ foram então estimados conforme González-Recio et al. (2007) e Gutiérrez et al. (2009), por meio da seguinte equação: $\Delta \mathrm{F}_{\mathrm{i}}=1-\sqrt[\mathrm{ECG}_{\mathrm{i}}-1]{1-\mathrm{F}_{\mathrm{i}}}$, em que $\mathrm{ECG}_{\mathrm{i}}$ é o número equivalente de gerações completas do animal.

A depressão por endogamia foi estimada para a PLDC durante a lactação. Utilizaram-se os controles entre o $5^{\circ}$ e o $305 .^{\circ}$ dias da lactação. As condições estabelecidas para a inclusão das vacas no estudo foram: idade ao parto entre 20 e 60 meses; primeiro controle realizado até 45 dias após o parto; no mínimo, três controles na lactação. As vacas foram distribuídas em oito subclasses de idade-estação de parto, que foram: duas estações, abril-setembro e outubro-março; e quatro classes de idade - 20-35, 36-47, 48-56 e 57-60 meses. Os grupos de contemporâneas (GC) foram formados por vacas cujas PLDC foram mensuradas no mesmo rebanho, ano e mês, tendo sido descartadas aquelas informações pertencentes aos GC com menos de três animais. Os registros de PLDC foram distribuídos em subclasses de rebanho-período de parto (RA), com 12 períodos de dois anos cada (1989-1990, ..., 2012-2013). As subclasses RA com 
menos de cinco observações foram eliminadas da análise. Com o objetivo de obter estimativas mais acuradas do efeito da endogamia, somente se utilizaram registros fenotípicos de animais cujo ECG era maior ou igual a dois (Gutiérrez et al., 2009). Os animais foram ainda distribuídos em quatro classes de $\Delta \mathrm{F}_{\mathrm{i}}: \mathrm{C} 0$, $\Delta \mathrm{F}_{\mathrm{i}}=0 \% ; \mathrm{C} 1,0 \%<\Delta \mathrm{F}_{\mathrm{i}} \leq 1,7861 \% ; \mathrm{C} 2,1,7861 \%<\Delta \mathrm{F}_{\mathrm{i}}$ $\leq 3,6603 \%$; $\mathrm{C} 3, \Delta \mathrm{F}_{\mathrm{i}}>3,6603 \%$. Na escala de $\mathrm{F}_{\mathrm{i}}$ para o ECG médio das vacas com informação fenotípica $(4,581)$, as classes equivalentes seriam: $0 \% ; 0 \%<\mathrm{F}_{\mathrm{i}} \leq$ $6,25 \% ; 6,25 \%<\mathrm{F}_{\mathrm{i}} \leq 12,50 \% ; \mathrm{F}_{\mathrm{i}}>12,50 \%$. A estrutura do conjunto de dados após as restrições é apresentada na Tabela 1 e nas Figuras 1 e 2. A matriz de parentesco utilizada foi formada por 25.913 animais.

A depressão por endogamia, na PLDC, foi estimada pela inclusão do efeito de $\Delta \mathrm{F}_{\mathrm{i}}$ no modelo de regressão aleatória (M1), conforme descrito a seguir:

$$
\begin{aligned}
& \mathrm{PLDC}_{\mathrm{ijklmv}}=\mathrm{GC}_{\mathrm{i}}+\mathrm{IE}_{\mathrm{j}}+\mathrm{FO}_{\mathrm{k}}+\mathrm{C} \Delta \mathrm{F}_{\mathrm{l}} \\
& +\sum_{\mathrm{o}=1}^{4} \mathrm{RA}_{\mathrm{mo}} \phi_{\mathrm{o}}(\mathrm{d})+\sum_{\mathrm{o}=1}^{4} \pm_{\mathrm{vo}} \phi_{\mathrm{o}}(\mathrm{d})+\sum_{\mathrm{o}=1}^{4} \mathrm{p}_{\mathrm{vo}} \phi_{\mathrm{o}}(\mathrm{d})+\mathrm{e}_{\mathrm{ijklmv}},
\end{aligned}
$$

em que: $\mathrm{GC}_{\mathrm{i}}$ é o efeito do i-ésimo grupo contemporâneo; $\mathrm{IE}_{\mathrm{j}}$ é o efeito da j-ésima subclasse de idade-estação do parto, aninhada na classe de dias em lactação (20 classes de 15 dias: 5-19, ..., 289-305); $\mathrm{FO}_{\mathrm{k}}$ é o efeito da k-ésima frequência de ordenha $(\mathrm{k}=1,2$ ou 3$)$ no dia do controle, aninhada na classe de dias em lactação (20 classes de 15 dias: $5-19, \ldots, 289-305) ; C \Delta \mathrm{F}_{1}$ é o efeito da l-ésima classe de $\Delta \mathrm{F}_{\mathrm{i}}$, como definida anteriormente, aninhada na classe de dias em lactação (20 classes de 15 dias: $5-19, \ldots, 289-305) ; \mathrm{RA}_{\text {mo }}$ é o o-ésimo coeficiente de regressão (efeito fixo) específico para a m-ésima subclasse de rebanho-período de parto; $\alpha_{\mathrm{vo}}$ e $p_{\mathrm{vo}}$ são os o-ésimos coeficientes de regressão, para os efeitos aleatórios genético-aditivo e de ambiente permanente

Tabela 1. Estatísticas descritivas do banco de dados após filtros e consistências.

\begin{tabular}{lcccc}
\hline Variável & Média & $\mathrm{DP}^{(1)}$ & Mínimo & Máximo \\
\hline Observações & 89.490 & - & - & - \\
Vacas em observação & 11.675 & - & - & - \\
Vacas por rebanho por ano & 10,5 & 11,1 & 1 & 117 \\
Observações/GC(2) & 9,7 & 8,5 & 3 & 80 \\
Observações/RA $/ \mathrm{RA}^{(3)}$ & 121,9 & 137,8 & 5 & 1151 \\
$\begin{array}{l}\text { Produção de leite no dia } \\
\text { do controle }(\mathrm{kg})\end{array}$ & 13,2 & 6,6 & 0,1 & 42,1 \\
\hline
\end{tabular}

${ }^{(1)}$ Desvio-padrão. ${ }^{(2)} \mathrm{GC}$, grupo de contemporâneas formado por rebanho, ano e mês do controle. ${ }^{(3)} \mathrm{RA}$, rebanho-período de parto. da vaca v; $\varphi_{0}(\mathrm{~d})$ é o o-ésimo polinômio ortogonal de Legendre, correspondente ao dia d da lactação; e $\mathrm{e}_{\mathrm{ijklmv}}$ é o resíduo aleatório. Para as regressões utilizaram-se os polinômios ortogonais de Legendre cúbicos.

Emnotação matricial, o modelo pode ser representado como: $\mathrm{Y}=\mathrm{Xb}+\mathrm{Za}+\mathrm{Wp}+\mathrm{e}$, em que: $\mathrm{Y}$ é o vetor das observações; $b$, o vetor dos efeitos sistemáticos (GC, IE, FO, $\mathrm{C} \Delta \mathrm{F}$ e RA); a, o vetor dos efeitos aleatórios genético-aditivos; $\mathrm{p}$, o vetor dos efeitos aleatórios de ambiente permanente; e é o vetor dos resíduos aleatórios; e X, Z e W são as matrizes de incidência, correspondentes às observações quanto aos efeitos sistemáticos, efeitos aleatórios genético-aditivos e de ambiente permanente, respectivamente. Para este modelo, assumiram-se as seguintes pressuposições:

$$
\begin{aligned}
& \mathrm{y} \mid \mathrm{b}, \mathrm{a}, \mathrm{p}, \mathrm{R} \sim \mathrm{NMV}(\mathrm{Xb}+\mathrm{Za}+\mathrm{Wp}, \mathrm{R}), \\
& \operatorname{Var}\left[\begin{array}{l}
\mathrm{a} \\
\mathrm{p} \\
\mathrm{e}
\end{array}\right]=\left[\begin{array}{ccc}
\Lambda_{\mathrm{A}} \otimes \mathrm{A} & 0 & 0 \\
0 & \Lambda_{\mathrm{P}} \otimes \mathrm{I} & 0 \\
0 & 0 & \mathrm{R}
\end{array}\right],
\end{aligned}
$$

em que: $\Lambda_{\mathrm{A}}$ e $\Lambda_{\mathrm{p}}$ são, respectivamente, as matrizes de covariâncias genético-aditivas e de ambiente permanente entre os coeficientes de regressão; $\mathrm{R}$ é a matriz de variâncias residuais, que é uma matriz diagonal com componentes de variância específicos para os intervalos 5-30, 31-60, 61-120, 121-270 e 271-305 dias em lactação, conforme descrito por Pereira et al. (2013b). Além disso, assumiu-se que os resíduos para diferentes dias da lactação eram não correlacionados. A é a matriz do numerador dos

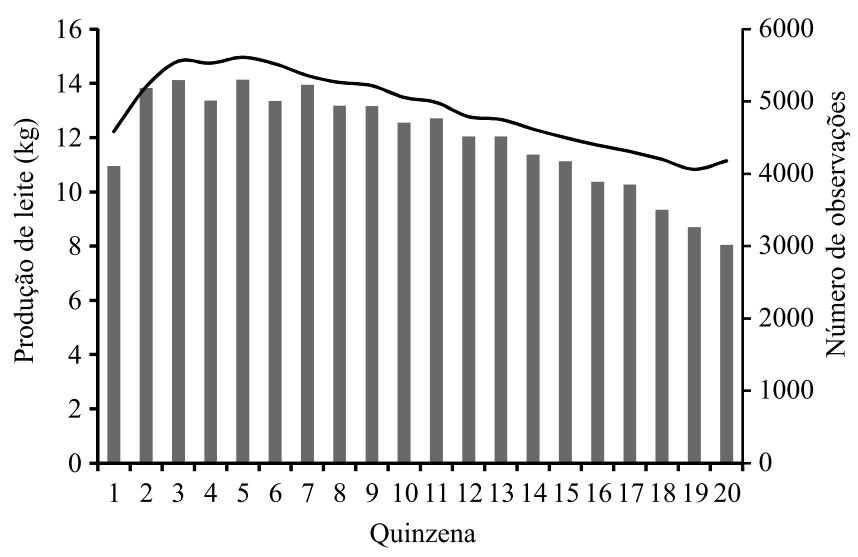

Figura 1. Número de observações (barras) e média da produção de leite, no dia do controle (linha), de acordo com a quinzena da lactação. 
coeficientes de parentesco entre os animais; I é uma matriz de identidade, e $\otimes$ é o operador produto direto entre matrizes.

A análise foi realizada por método bayesiano, com o programa GIBBS3F90 (Misztal et al., 2015). As distribuições a priori, assumidas para os parâmetros do modelo, foram: $b \propto$ constante,

$$
\begin{aligned}
& \mathrm{a}\left|\Lambda_{\mathrm{A}} \sim \operatorname{NMV}\left(0, \Lambda_{\mathrm{A}} \otimes \mathrm{A}\right), \mathrm{p}\right| \Lambda_{\mathrm{P}} \sim \operatorname{NMV}\left(0, \Lambda_{\mathrm{P}} \otimes \mathrm{I}\right), \\
& \Lambda_{\mathrm{A}}\left|\mathrm{v}_{\mathrm{a}}, \mathrm{S}_{\mathrm{a}} \sim \mathrm{WI}\left(\mathrm{v}_{\mathrm{a}}, \mathrm{v}_{\mathrm{a}} \mathrm{S}_{\mathrm{a}}\right), \Lambda_{\mathrm{P}}\right| \mathrm{v}_{\mathrm{p}}, \mathrm{S}_{\mathrm{p}} \sim \mathrm{WI}\left(\mathrm{v}_{\mathrm{p}}, \mathrm{v}_{\mathrm{p}} \mathrm{S}_{\mathrm{p}}\right),
\end{aligned}
$$

$\mathrm{R} \mid \mathrm{v}_{\mathrm{e}}, \mathrm{S}_{\mathrm{e}} \sim \mathrm{WI}\left(\mathrm{v}_{\mathrm{e}}, \mathrm{v}_{\mathrm{e}} \mathrm{S}_{\mathrm{e}}\right)$, em que: NMV e WI são indicativos das distribuições normal multivariada $\mathrm{e}$ Wishart invertida, respectivamente; e $\mathrm{v}_{\mathrm{a}}, \mathrm{S}_{\mathrm{a}}, \mathrm{v}_{\mathrm{p}}, \mathrm{S}_{\mathrm{p}}$ e $\mathrm{V}_{\mathrm{c}}, \mathrm{S}_{\mathrm{c}}$ representam o grau de confiança e os valores a priori para as matrizes de variâncias e covariâncias genético-aditivas, de ambiente permanente e residuais, respectivamente.

Para a avaliação do impacto da consideração ou não do efeito da endogamia sobre a avaliação genética dos animais, para a produção de leite e persistência desta durante a lactação, uma segunda análise foi realizada com um modelo estatístico M0, semelhante ao $\mathrm{M} 1$, mas sem se considerar o efeito de $\Delta \mathrm{F}_{\mathrm{i}}$. Para a comparação dos modelos, utilizaram-se os seguintes critérios: correlação $(\mathrm{R})$ entre o fenótipo predito com o respectivo modelo e o fenótipo observado; percentual de viés quadrático (PSB), conforme Ali \& Schaeffer (1987); quadrado médio do resíduo (QMR), critério de informação da deviance (DIC), conforme proposto por Spiegelhalter et al. (2002); e estimativas de parâmetros genéticos. Uma diferença de pelo menos sete unidades entre os valores de DIC dos modelos M0 e M1 foi considerada importante (Spiegelhalter et al., 2002). Ao se utilizar M0 ou M1, as correlações de ordem entre os valores genéticos preditos foram estimadas para os seguintes coeficientes de regressão aleatória: o primeiro, $\alpha_{1}$, associado ao nível médio de produção durante a lactação; e o segundo, $\alpha_{2}$, associado à persistência da produção durante a lactação. Adicionalmente, quantificaram-se as mudanças de ordem de classificação (baseada nos valores genéticos preditos para $\alpha_{1}$ e $\alpha_{2}$ ), de acordo com as classes de $\Delta F_{i}$ (como definidas anteriormente), quando se utilizaram os modelos M0 ou M1.

As inferências sobre os parâmetros de interesse foram realizadas a partir de suas correspondentes distribuições marginais a posteriori. Obteve-se uma cadeia de $500 \mathrm{mil}$ amostras para cada modelo (M0 ou M1). Após o descarte das primeiras 50 mil amostras (burn-in), realizou-se o intervalo de descarte amostral de 10 , tendo restado 45 mil amostras, em cuja base se realizaram as inferências. A convergência foi monitorada pela inspeção gráfica das amostras $\times$ iterações e, também, pelos critérios de Heidelberger \& Welch (1983) e Geweke (1992), os quais foram calculados com o auxílio do pacote BOA do R (Smith, 2007).

Com as médias a posteriori das estimativas de depressão endogâmica (soluções para o efeito $\mathrm{C} \Delta \mathrm{F}$ ), realizou-se a verificação da existência de variações na depressão endogâmica em função da classe de endogamia (classe de $\Delta \mathrm{F}_{\mathrm{i}}$ ) e da fase da lactação. As soluções para as classes de $\Delta \mathrm{F}_{\mathrm{i}} \mathrm{C} 1, \mathrm{C} 2$ e $\mathrm{C} 3$ foram expressas como diferenças (DIF) em relação à classe $\mathrm{C} 0\left(\Delta \mathrm{F}_{\mathrm{i}}=0 \%\right)$. O modelo $\left(\mathrm{M}_{\mathrm{INT}}\right)$ utilizado na análise desta etapa foi definido como

$$
\mathrm{Y}_{\mathrm{ijk}}=\mu+\mathrm{C} \Delta \mathrm{F}_{\mathrm{i}}+\mathrm{FL}_{\mathrm{j}}+\mathrm{I}_{\mathrm{ij}}+\varepsilon_{\mathrm{ijk}}
$$

em que: $Y_{\mathrm{ij}}$ é a medida de depressão endogâmica, para a classe de endogamia i e fase da lactação j; $\mathrm{C} \Delta \mathrm{F}_{\mathrm{i}}$ e $\mathrm{FL}_{\mathrm{j}}$ são os efeitos fixos da classe de endogamia $\mathrm{i}(\mathrm{i}=1$, 2 ou 3) e da fase de lactação j ( $1=5-94 ; 2$ = 95-199; 3 = 200-305 dias); $\mathrm{I}_{\mathrm{ij}}$ é o efeito da interação classe de endogamia $\times$ fase da lactação; e $\varepsilon_{\mathrm{ijk}}$ é o erro aleatório. Para a execução da análise, utilizou-se o procedimento GLM do SAS (SAS Institute, Cary, NC, EUA).

\section{Resultados e Discussão}

A PLDC média durante a lactação variou entre 10,82 kg e 14,95 kg (Figura 1). O pico de produção ocorreu, em média, entre os dias 35 e 79 da lactação, mais tarde do que o relatado por Pereira et al. (2013a, 2013b), ao trabalharem com parte do mesmo conjunto de dados utilizados no presente trabalho, que incluíram observações de vacas leiteiras do cruzamento das raças Gir Leiteiro $\times$ Holandesa. Os autores encontraram que o número de informações disponíveis decresceu no último terço da lactação, provavelmente em razão da ocorrência de lactações curtas (menos que 200 dias) na raça. $\mathrm{O}$ percentual de observações nas classes de $\Delta \mathrm{F}_{\mathrm{i}}$ $\mathrm{C} 0$ e $\mathrm{C} 1$ foi semelhante, e a soma dessas duas classes representou, durante a lactação, aproximadamente 95\% do total de registros disponíveis (Figura 2). A última classe (C3) incluiu somente cerca de $2 \%$ dos dados, em qualquer estádio da lactação. Informações 
detalhadas sobre a estrutura e a variabilidade genética da população aqui estudada podem ser encontradas em Santana Jr. et al. (2014).

As soluções para a depressão por endogamia (DIF), de acordo com a classe de $\Delta \mathrm{F}_{\mathrm{i}}$ e a quinzena da lactação variaram entre -1,238 e -0,135 kg (Figura 3), o que indica redução da produção de leite com o aumento da endogamia. Quando se consideram as médias das soluções de depressão por endogamia, durante a lactação e um período de lactação de 305 dias, esperam-se reduções de 80 a $250 \mathrm{~kg}$ de leite na produção acumulada, a depender do nível de endogamia da vaca. $\mathrm{Na}$ análise das DIF que utilizou o modelo $\mathrm{M}_{\mathrm{INT}}$, o coeficiente de determinação foi 0,81 . Houve interação entre a classe de $\Delta \mathrm{F}_{\mathrm{i}}$ e a fase da lactação. Nas DIF analisadas dentro de cada fase da lactação (Tabela 2), observaram-se diferenças entre as classes de $\Delta \mathrm{F}_{\mathrm{i}}$. Nas duas primeiras fases da lactação (5 a 199 dias), as DIF para a primeira classe de $\Delta \mathrm{F}_{\mathrm{i}}(\mathrm{C} 1)$ foram de magnitude inferior às DIF para as duas classes de $\Delta \mathrm{F}_{\mathrm{i}}$ subsequentes ( $\mathrm{C} 2 \mathrm{e} \mathrm{C} 3$ ), que não diferiram entre si. Na terceira fase da lactação (200 a 305 dias), as DIF para as 3 classes de $\Delta F_{i}$ diferiram entre si, com incremento da depressão da produção de leite à medida que $\Delta \mathrm{F}_{\mathrm{i}}$ aumentou.

Nas classes de $\Delta F_{i} C 1$ e $C 2$, não se observaram diferenças entres as DIF para as respectivas fases da lactação (Tabela 2), o que indica que a depressão da produção de leite, em decorrência do incremento da endogamia, é de mesma magnitude durante a lactação para essas duas classes de $\Delta \mathrm{F}_{\mathrm{i}} \mathrm{e}$, como consequência, $\mathrm{o}$ nível de produção das vacas éreduzido, mas a persistência

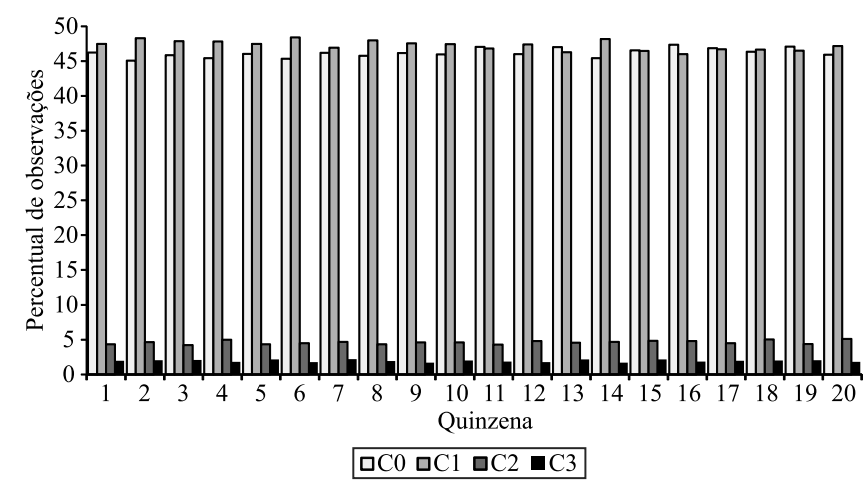

Figura 2. Percentual de observações de acordo com a quinzena da lactação e a classe de incremento individual de endogamia $\left(\Delta \mathrm{F}_{\mathrm{i}}\right)$, em que: $\mathrm{C} 0, \Delta \mathrm{F}_{\mathrm{i}}=0 \% ; \mathrm{C} 1,0 \%<\Delta \mathrm{F}_{\mathrm{i}}$ $\leq 1,7861 \%$; $22,1,7861 \%<\Delta \mathrm{F}_{\mathrm{i}} \leq 3,6603 \% ; \mathrm{C} 3, \Delta \mathrm{F}_{\mathrm{i}}>3,6603 \%$. da produção de leite não é alterada. Contudo, na última classe de $\Delta \mathrm{F}_{\mathrm{i}}(\mathrm{C} 3)$, as DIF para as duas últimas fases da lactação ( 95 a 305 dias) foram de magnitude superior às DIF para a primeira fase da lactação (5 a 94 dias). Isto é uma indicação de que, em $\mathrm{C} 3$, além da redução no nível de produção das vacas, ocorre também a diminuição da persistência da produção de leite durante a lactação. Em C3, as DIF para as duas últimas fases da lactação não diferiram entre si.

Ao utilizar um modelo fixo e registros de produção no dia do controle de vacas Holandês nos EUA, Thompson et al. (2000a) verificaram, em geral, efeito negativo do incremento de $F_{i}$ sobre a produção de leite, especialmente para níveis de $F_{i}$ superiores a $7 \%$. No entanto, diferentemente dos resultados encontrados no presente trabalho, os autores obtiveram maiores estimativas de redução da produção no primeiro terço da lactação. Com o mesmo modelo de Thompson

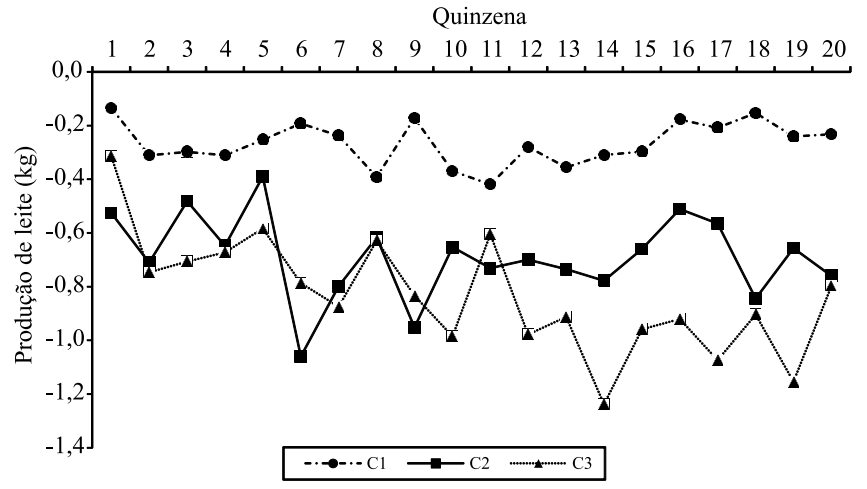

Figura 3. Soluções para o efeito da endogamia sobre a produção de leite no dia do controle, expressas como diferenças em relação à classe $\mathrm{C} 0, \Delta \mathrm{F}_{\mathrm{i}}=0 \%$, de acordo com a quinzena da lactação e a classe de incremento individual de endogamia $\left(\Delta \mathrm{F}_{\mathrm{i}}\right)$, em que: $\mathrm{C} 1,0 \%<\Delta \mathrm{F}_{\mathrm{i}} \leq 1,7861 \%$; 2 , $1,7861 \%<\Delta \mathrm{F}_{\mathrm{i}} \leq 3,6603 \% ; \mathrm{C} 3, \Delta \mathrm{F}_{\mathrm{i}}>3,6603 \%$.

Tabela 2. Médias (quadrados mínimos) e erros-padrão (entre parênteses) da depressão endogâmica sobre a produção de leite (kg por dia), de acordo com a classe de endogamia e a fase da lactação ${ }^{(1)}$.

\begin{tabular}{llll}
\hline Fase de & \multicolumn{3}{c}{ Classes de incremento individual de endogamia $\left(\Delta \mathrm{F}_{\mathrm{i}}\right)$} \\
\cline { 2 - 4 } lactação & $0 \%<\Delta \mathrm{F}_{\mathrm{i}} \leq 1,7861 \% 1,7861 \%<\Delta \mathrm{Fi} \leq 3,6603 \%$ & $\Delta \mathrm{F}_{\mathrm{i}}>3,6603 \%$ \\
\hline 5 a 94 dias & $-0,250(0,057) \mathrm{aA}$ & $-0,636(0,057) \mathrm{aB}$ & $-0,640(0,057) \mathrm{aB}$ \\
95 a 199 dias & $-0,317(0,052) \mathrm{aA}$ & $-0,741(0,052) \mathrm{aB}$ & $-0,837(0,052) \mathrm{aB}$ \\
200 a 305 dias & $-0,231(0,052) \mathrm{aA}$ & $-0,682(0,052) \mathrm{aB}$ & $-1,011(0,052) \mathrm{bC}$ \\
\hline
\end{tabular}

${ }^{(1)}$ Médias seguidas por letras iguais, minúsculas nas colunas e maiúsculas nas linhas, não diferem pelo teste Tukey, a $5 \%$ de probabilidade. 
et al. (2000a), mas com dados da raça Jersey dos EUA, Thompson et al. (2000b) reportaram resultados semelhantes àqueles da raça Holandesa. Panetto et al. (2010) estudaram um rebanho da raça Guzerá e observaram efeito negativo do incremento de $F_{i}$ sobre a média da produção de leite do dia no controle das vacas. Os únicos dois estudos sobre a raça Gir encontrados na literatura verificaram o impacto de $F_{i}$ sobre as estimativas de produção acumulada em até 305 dias de lactação (P305). Reis Filho et al. (2015) utilizaram registros de P305 da raça Gir e relataram efeito quadrático de $\mathrm{F}_{\mathrm{i}}$ sobre a produção de leite, com redução do desempenho para qualquer nível de $F_{i}$. Queiroz et al. (1993) observaram efeito linear de $F_{i}$ sobre P305 (-18.99 kg/1\% de incremento em $\left.F_{i}\right)$ da raça Gir.

Os valores de QMR, PSB e R, para os modelos M0 e M1, foram 1,591041 e 1,591018, 12,042920 e $12,042740,0,9814258$ e 0,9814260 , respectivamente. De acordo com os critérios de comparação de modelos utilizados, uma pequena diferença foi identificada a favor do modelo M1, que considerou o efeito da endogamia sobre a PLDC. Os valores de DIC diferiram entre os modelos, tendo-se identificado uma diferença de 2.524 unidades a favor do modelo M1. Esses resultados são indicativos de que considerar o efeito da endogamia, no modelo de avaliação genética, permite que este se ajuste um pouco melhor aos dados.

As médias a posteriori das estimativas de variâncias genético-aditivas, de ambiente permanente e residuais, bem como as de herdabilidades e correlações genéticas foram praticamente idênticas entre os dois modelos, durante a lactação (Figuras 4 e 5), o que indica que a inclusão ou não do efeito da endogamia no modelo não afeta as estimativas de componentes de variância e demais parâmetros genéticos. Ferreira et al. (1999) comparam modelos com ou sem o efeito fixo de $F_{i}$, sobre características de crescimento de bovinos da raça Hereford, e concluíram que não houve diferenças entre as estimativas de herdabilidade ao compararem os modelos. As estimativas de herdabilidade variaram entre 0,17 e 0,22 e foram semelhantes às reportadas por Pereira et al. (2013a, 2013b), que trabalharam com parte do conjunto de dados utilizados no presente estudo.

As correlações de ordem para touros (pais de vacas com registros de produção) e vacas com registros de produção, quando classificados pelos valores genéticos preditos para os coeficientes de regressão $\alpha_{1}$ e $\alpha_{2}$ foram sempre superiores a 0,9999 . As alterações das avaliações genéticas e respectivas classificações dos animais para $\alpha_{1}$ e $\alpha_{2}$, quando o modelo M0 foi substituído por M1, foram, em média, mínimas ou nulas (Tabela 3). Um discreto aumento dos valores genéticos preditos para $\alpha_{1}$ foi observado com o incremento do nível de endogamia das vacas, tendência não observada para $\alpha_{2}$. Para $\alpha_{1}$, nas classes de endogamia C2 e C3, observou-se, em média, uma discreta melhoria na classificação das vacas. Com estes resultados, pode-se inferir que quando há alterações na avaliação genética dos animais, decorrentes do uso de um modelo estatístico que considera a endogamia, essas alterações têm pouco ou nenhum impacto sobre a seleção dos

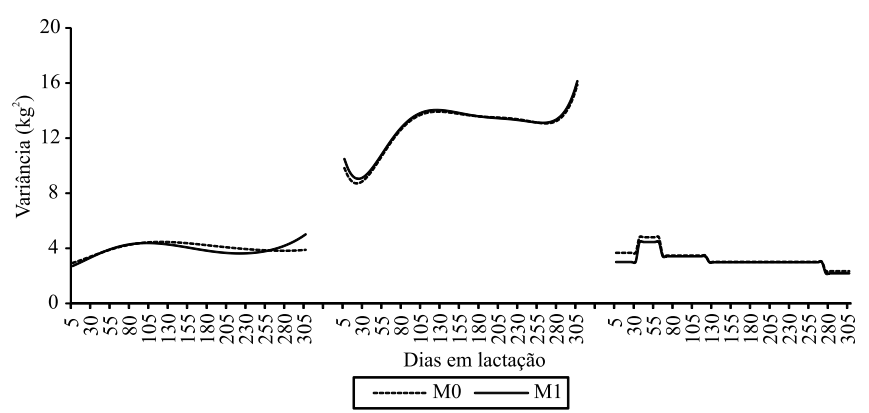

Figura 4. Estimativas médias a posteriori, para as variâncias genético-aditivas (esquerda), de ambiente permanente (centro) e residuais (direita), de acordo com o dia da lactação, obtidas com os modelos estatísticos M0 (sem o efeito do incremento individual de endogamia) e M1 (com o efeito do incremento individual de endogamia).

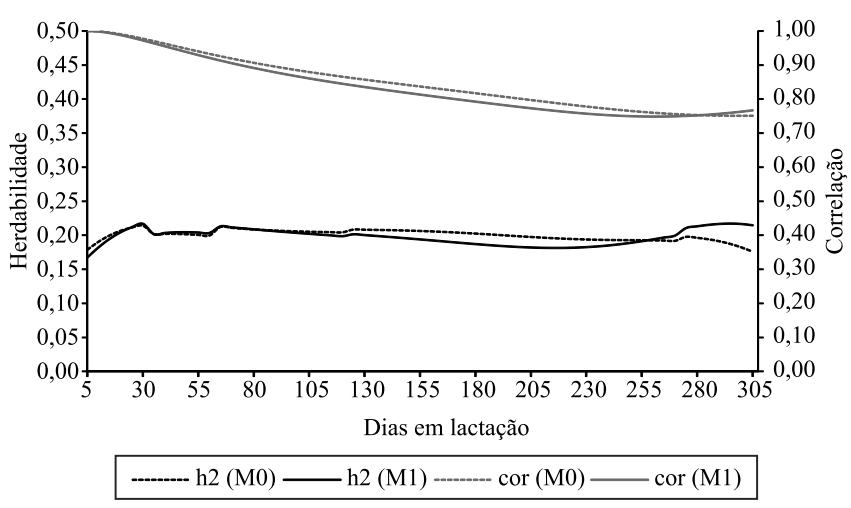

Figura 5. Estimativas médias a posteriori, para as herdabilidades (h2) e correlações (entre o dia 5 e os demais dias) genético-aditivas (cor) de acordo com o dia da lactação, obtidas com os modelos estatísticos M0 (sem o efeito do incremento individual de endogamia) e M1 (com o efeito do incremento individual de endogamia). 
Tabela 3. Alterações das classificações (CLAS) e dos valores genéticos preditos (VG, $\mathrm{kg})$ para o primeiro $\left(\alpha_{1}\right)$ e o segundo $\left(\alpha_{2}\right)$ coeficientes de regressão aleatória, entre modelos de avaliação genética sem e com o efeito da endogamia.

\begin{tabular}{|c|c|c|c|c|c|c|c|c|c|c|c|c|}
\hline \multirow[t]{2}{*}{ Classificações } & \multicolumn{6}{|c|}{ Alteração ( $\alpha_{1}-$ nível médio de produção) } & \multicolumn{6}{|c|}{ Alteração $\left(\alpha_{2}-\right.$ persistência da produção $)$} \\
\hline & Mínimo & Quartil 1 & Mediana & Média & Quartil 3 & Máximo & Mínimo & Quartil 1 & Mediana & Média & Quartil 3 & Máximo \\
\hline \multicolumn{13}{|c|}{ Vacas (CLAS) } \\
\hline $\mathrm{C} 0$ & -2 & 0 & 0 & 0 & 0 & 3 & -1 & 0 & 0 & 0 & 0 & 1 \\
\hline $\mathrm{C} 1$ & -4 & 0 & 0 & 0 & 0 & 2 & -1 & 0 & 0 & 0 & 0 & 2 \\
\hline $\mathrm{C} 2$ & -5 & -2 & -1 & -1 & -1 & 1 & -1 & 0 & 0 & 0 & 0 & 2 \\
\hline $\mathrm{C} 3$ & -10 & -3 & -2 & -2 & -1 & 3 & -2 & 0 & 0 & 0 & 1 & 3 \\
\hline \multicolumn{13}{|l|}{ Vacas (VG) } \\
\hline $\mathrm{C} 0$ & $-0,225$ & 0,000 & 0,017 & 0,020 & 0,036 & 0,254 & $-0,007$ & 0,000 & 0,000 & 0,000 & 0,001 & 0,006 \\
\hline $\mathrm{C} 1$ & $-0,140$ & 0,007 & 0,025 & 0,034 & 0,053 & 0,373 & $-0,008$ & $-0,001$ & 0,000 & 0,000 & 0,001 & 0,005 \\
\hline $\mathrm{C} 2$ & $-0,014$ & 0,109 & 0,167 & 0,177 & 0,226 & 0,460 & $-0,014$ & $-0,002$ & $-0,001$ & $-0,001$ & 0,000 & 0,004 \\
\hline $\mathrm{C} 3$ & $-0,286$ & 0,157 & 0,252 & 0,249 & 0,308 & 1,112 & $-0,044$ & $-0,004$ & $-0,002$ & $-0,003$ & 0,000 & 0,014 \\
\hline \multicolumn{13}{|l|}{ Touros } \\
\hline CLAS & -6 & 0 & 0 & 0 & 1 & 3 & -2 & 0 & 0 & 0 & 0 & 2 \\
\hline VG & $-0,177$ & 0,002 & 0,036 & 0,050 & 0,078 & 0,565 & $-0,022$ & $-0,001$ & 0,000 & 0,000 & 0,001 & 0,008 \\
\hline
\end{tabular}

Valores negativos de CLAS indicam que a classificação dos animais melhorou, quando se utilizou, para a avaliação genética, o modelo estatístico com o efeito da endogamia. $\mathrm{C} 0, \Delta \mathrm{F}_{\mathrm{i}}=0 \% ; \mathrm{C} 1,0 \%<\Delta \mathrm{F}_{\mathrm{i}} \leq 1,7861 \% ; \mathrm{C} 2,1,7861 \%<\Delta \mathrm{F}_{\mathrm{i}} \leq 3,6603 \% ; \mathrm{C} 3, \Delta \mathrm{F}_{\mathrm{i}}>3,6603 \%$.

animais na população. Resultados semelhantes foram observados em estudos com outras raças de bovinos leiteiros em diferentes países (Wiggans et al., 1995; Ruíz-Flores et al., 2011). No entanto, Wiggans et al. (1995), que estudaram o impacto de se considerar o $F_{i}$ nas avaliações genéticas nacionais das raças leiteiras nos EUA, salientaram a importância de se levar em conta tal efeito, mesmo que o impacto sobre as avaliações genéticas dos animais tenha sido pequeno. Os autores ressaltaram duas questões importantes para tal recomendação: o incremento da endogamia nas populações é inevitável e, então, o impacto do ajuste para $\mathrm{F}_{\mathrm{i}}$, sobre as predições de valores genéticos dos animais, poderia se tornar maior no futuro; avaliações iniciais de touros jovens (poucas filhas) poderiam ser subestimadas, quando sua progênie fosse proveniente de acasalamentos com vacas mais aparentadas com esses touros.

Os resultados obtidos evidenciaram o efeito prejudicial da endogamia sobre a produção de leite, possivelmente ocasionado pela redução da eficiência fisiológica das vacas leiteiras. Santana Jr. et al. (2014) observaram incremento do coeficiente de coancestralidade médio, nos últimos anos, para a raça Gir. O incremento deste parâmetro está relacionado ao aumento das futuras taxas de endogamia, pois, o coeficiente de coancestralidade dos pais é igual ao coeficiente de endogamia de sua progênie (Falconer \& Mackay, 1996). Portanto, para minimizar os impactos negativos da endogamia, como a depressão endogâmica e a redução da variabilidade genética, há necessidade de gerenciamento da endogamia no Programa Nacional de Melhoramento do Gir Leiteiro.

\section{Conclusões}

1. O incremento da endogamia tem um impacto negativo sobre a produção de leite e, quando em níveis mais elevados, sobre a persistência da produção de leite na lactação.

2. A inclusão do efeito da endogamia no modelo estatístico de avaliação genética da raça Gir Leiteiro para a produção de leite, não afeta as estimativas de parâmetros genéticos e, praticamente, não altera a avaliação genética dos animais e suas classificações.

\section{Agradecimentos}

À Associação Brasileira dos Criadores de Gir Leiteiro, por permitir o acesso ao banco de dados histórico da raça.

\section{Referências}

ALI, T.E.; SCHAEFFER, L.R. Accounting for covariances among test day milk yields in dairy cows. Canadian Journal of Animal Science, v.67, p.637-644, 1987. DOI: 10.4141/cjas87-067. 
FALCONER, D.S.; MACKAY, T.F.C. Introduction to Quantitative Genetics. $4^{\text {th }}$ ed. Essex: Longman, 1996. 464p.

FERREIRA, G.B.; MACNEIL, M.D.; VAN VLECK, L.D. Variance components and breeding values for growth traits from different statistical models. Journal of Animal Science, v.77, p.2641-2650, 1999.

GEWEKE, J. Evaluating the accuracy of sampling-based approaches to the calculation of posterior moments. In: BERNARDO, J.M.; BERGER, J.O.; DAWID, A.P.; SMITH, A.F. M. (Ed.). Bayesian Statistics 4. Oxford: Oxford University Press, 1992. p.625-631.

GONZÁLEZ-RECIO, O.; LÓPEZ DE MATURANA, E.; GUTIÉRREZ, J.P. Inbreeding depression on female fertility and calving ease in Spanish dairy cattle. Journal of Dairy Science, v.90, p.5744-5752, 2007. DOI: 10.3168/jds.2007-0203.

GUTIÉRREZ, J.P.; CERVANTES, I.; GOYACHE, F. Improving the estimation of realized effective population sizes in farm animals. Journal of Animal Breeding and Genetics, v.126, p.327-332, 2009. DOI: 10.1111/j.1439-0388.2009.00810.x.

HANSEN, P.J. Physiological and cellular adaptations of zebu cattle to thermal stress. Animal Reproduction Science, v.82/83, p.349-360, 2004. DOI: 10.1016/j.anireprosci.2004.04.011.

HEIDELBERGER, P.; WELCH, P. Simulation run length control in the presence of an initial transient. Operations Research, v.31, p.1109-1144, 1983. DOI: 10.1287/opre.31.6.1109.

JONSSON, N.N. The productivity effects of cattle tick (Boophilus microplus) infestation on cattle, with particular reference to Bos indicus cattle and their crosses. Veterinary Parasitology, v.137, p.1-10, 2006. DOI: 10.1016/j.vetpar.2006.01.010.

MISZTAL, I.; TSURUTA, S.; LOURENÇO, D.; AGUILAR, I.; LEGARRA, A.; VITEZICA, Z. Manual for BLUPF90 family of programs. Disponível em: < http://nce.ads.uga.edu/wiki/lib/exe/ fetch.php?media=blupf90_all2.pdf $>$. Acesso em: 7 jul. 2015.

PANETTO, J.C. do C.; VERNEQUE, R. da S.; PEIXOTO, M.G.C.D.; BRUNELI, F.A.T.; MACHADO, M.A.; MARTINS, M.F.; SILVA, M.V.G.B. da; ARBEX, W.A.; REIS; D.R. de L.; GERALDO, C.C.; MACHADO, C.H.C.; PEREIRA, M.A.; HORTOLANI, B.; VERCESI FILHO, A.E.; MACIEL, R. da S.; FERNANDES, A.R. Programa Nacional de Melhoramento do Gir Leiteiro: Sumário Brasileiro de Touros: Resultado do Teste de Progênie: $6^{\text {a }}$ prova de pré-seleção de touros: maio 2015. Juiz de Fora: Embrapa Gado de Leite, 2015. 82p. (Documentos, 177).

PANETTO, J.C.C.; GUTIÉRREZ, J.P.; FERRAZ, J.B.S.; CUNHA, D.G.; GOLDEN, B.L. Assessment of inbreeding depression in a Guzerat dairy herd: effects of individual increase in inbreeding coefficients on production and reproduction. Journal of Dairy Science, v.93, p.4902-4912, 2010. DOI: 10.3168/jds.2010-3197.

PEREIRA, R.J.; AYRES, D.R.; EL FARO, L.; VERCESI FILHO, A.E.; VERNEQUE, R. da S.; ALBUQUERQUE, L.G. de. Genetic parameters for production traits of dairy Gyr (Bos indicus $) \times$ Holstein cattle estimated with a random regression model. Livestock Science, v.158, p.24-31, 2013a. DOI: 10.1016/j. livsci.2013.10.003.
PEREIRA, R.J.; BIGNARDI, A.B.; EL FARO, L.; VERNEQUE, R.S.; VERCESI FILHO, A.E.; ALBUQUERQUE, L.G. Random regression models using Legendre polynomials or linear splines for test-day milk yield of dairy Gyr (Bos indicus) cattle. Journal of Dairy Science, v.96, p.565-574, 2013b. DOI: 10.3168/ jds.2011-5051.

QUEIROZ, S.A. de; LÔBO, R. B.; MARTINEZ, M.L. Efeito da endogamia sobre algumas características de importância econômica na raça Gir. Revista Brasileira de Zootecnia, v.22, p.773-786, 1993.

REIS FILHO, J.C.; VERNEQUE, R. da S.; TORRES, R. de A.; LOPES, P.S.; RAIDAN, F.S.S.; TORAL, F.L.B. Inbreeding on productive and reproductive traits of dairy Gyr cattle. Revista Brasileira de Zootecnia, v.44, p.174-179, 2015. DOI: 10.1590/ S1806-92902015000500002.

RUÍZ-FLORES, A.; GARCÍA-MUNGUÍA, C.A.; NÚÑEZ-DOMÍNGUEZ, R.; RAMÍREZ-VALVERDE， R.; LÓPEZ-ORDAZ， R.; GARCÍA-MUÑIZ，J.G. Inclusión del coeficiente de consanguinidad en los modelos de evaluación genética de bovinos Jersey y Suizo Americano en México. Revista Mexicana de Ciencias Pecuarias, v.2, p.381-391, 2011.

SANTANA JR., M.L.; PEREIRA, R.J.; BIGNARDI, A.B.; EL FARO, L.; TONHATI, H.; ALBUQUERQUE, L.G. History, structure, and genetic diversity of Brazilian Gir cattle. Livestock Science, v.163, p.26-33, 2014. DOI: 10.1016/j.livsci.2014.02.007.

SMITH, B.J. Boa: an R package for MCMC output convergence assessment and posterior inference. Journal of Statistical Software, v.21, p.1-37, 2007. DOI: 10.18637/jss.v021.i11.

SPIEGELHALTER, D.J.; BEST, N.G.; CARLIN, B.P.; LINDE, A. van der. Bayesian measures of model complexity and fit. Journal of the Royal Statistical Society. Series B. Statistical Methodology, v.64, p.583-639, 2002. DOI: 10.1111/1467-9868.00353.

STRANDÉN, I.; VUORI, K. RelaX2: pedigree analysis programme. In: WORLD CONGRESS ON GENETICS APPLIED TO LIVESTOCK PRODUCTION, 8., Belo Horizonte. Proceedings. Belo Horizonte: Instituto Prociência, 2006. p.27-30.

THOMPSON, J.R.; EVERETT, R.W.; HAMMERSCHMIDT, N.L. Effects of inbreeding on production and survival in Holsteins. Journal of Dairy Science, v.83, p.1856-1864, 2000a. DOI: 10.3168/jds.S0022-0302(00)75057-0.

THOMPSON, J.R.; EVERETT, R.W.; WOLFE, C.W. Effects of inbreeding on production and survival in Jerseys. Journal of Dairy Science, v.83, p.2131-2138, 2000b. DOI: $10.3168 /$ jds. S0022-0302(00)75096-X.

WIGGANS, G.R.; VANRADEN, P.M.; ZUURBIER, J. Calculation and use of inbreeding coefficients for genetic evaluation of United States dairy cattle. Journal of Dairy Science, v.78, p.1584-1590, 1995. DOI: $10.3168 /$ jds.S0022-0302(95)76782-0.

WOOLLIAMS J.A.; BERG, P.; DAGNACHEW, B.S.; MEUWISSEN, T.H.E. Genetic contributions and their optimization. Journal of Animal Breeding and Genetics, v.132, p.89-99, 2015. DOI: $10.1111 /$ jbg. 12148 . 